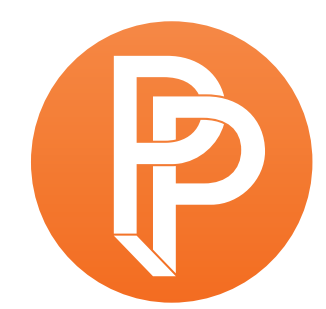

PERFORMANCE

PHILOSOPHY

\title{
COLLABORATION AS DIFFERENTIATION: RETHINKING INTERACTION INTRA-ACTIVELY
}

\author{
JOHN MACCALLUMINRIA, CNRS, UNIVERSITE PARIS-SACLAY \\ TEOMANACCARATO CENTRE FOR DANCE RESEARCH, COVENTRY UNIVERSITY
}

Wherever you are, imagine three other people sharing the space with you. They are seated on the floor,

legs crossed, backs straight, eyes closed, hands on knees, wearing loose-fitting skin that is not their own. They are breathing slowly and calmly but deeply and audibly. They are wearing headphones that indicate when to inhale and when to exhale. Their breath is regular; they take precisely six seconds to fill their lungs to capacity, and then another six seconds to completely void their lungs of air. Take a few breaths with them...in for six seconds...out for six seconds...in...out. It's ok if the imposed regularity of the clock is uncomfortable, it's supposed to be. This is not normal involuntary breathing, nor is it Pranayama, nor is it recovery from physical exertion. It's an exercise in aligning a bodily process to one of mechanical regularity. 


\section{PRELUDE: CHOREOGRAPHY, COMPOSITION, AND INTERACTION}

As composer-choreographer, we create work for stage and installation that is often described as "interactive performance," perhaps owing to our use of technologies for biosensing and motiontracking. Through our appropriation, use, and misuse of technologies from other disciplines, we have become implicated in cross-disciplinary discourse regarding interaction, and interaction design. We must acknowledge from the outset, however, that we do not believe there is such a thing as performance without interaction, or performance without technology. We employ the terms "interaction" and "technology" in their broadest senses and with intentional ambiguity to evoke the multiplicity of meanings they perform in different disciplinary contexts (music, dance, computer science, physics, chemistry, statistics). This paper is an attempt to interrogate assumptions regarding interaction within our own and other disciplines as a way of caring for our relationship to these fields. Our interest here is not to define interaction, nor to challenge or critique existing interpretations of the term. We also do not desire a common language across disciplines, such that we may design interaction together in a way that we all appear to understand, at the expense of collapsing the specificity of our different perspectives. As choreographercomposer traversing disciplinary cultures, what we are after, really, are strategies for interdisciplinary collaboration that resist subjugation of viewpoints and that not only tolerate, but rather require critical difference between practices to thrive.

We begin by arguing that the concept of "interaction" can only take on meaning in a situated context and is therefore an object subject to design. We look to Karen Barad's notion of "intraaction" as a way of framing the process of constructing concepts such as "interaction" and "things that interact," as well as the concept of "intra-action" itself (Barad 2007). From that viewpoint, we then discuss Susan Leigh Star's concept of "boundary objects," which, since its introduction in 1989, has become an important tool in the analysis of collaborative work. One of our goals in this discussion is to better understand how the concept of boundary objects relates to consensus and difference. Finally, we propose a practice of "critical appropriation" in which we assert that all use of technology (broadly construed) involves appropriation-an act of taking and making our own. Sustained attention to the ongoing act of appropriation, always already underway through use, is a practice of care for the multiplicity of that which is being used, and more generally, a practice of care for difference.

Move closer to one of the breathers, and sit down on the floor so that your knees are almost touching theirs. Really try to match the regularity of the breathing of the person directly in front of you. Now reach into the chest of this person. Through the skin that is not theirs, between the bones, and find their heart. Hold it with both hands as you continue to synchronize your breath with theirs. 


\section{HAUNTED BOUNDARIES [OF OBJECTS]}

Composers, choreographers, architects, engineers, city planners, human computer interaction designers, all construct technologies (public spaces, machines, software, choreography, buildings, compositions) that restrict and encourage different types of behavior in time. Across these and myriad other disciplinary practices, the approach of any designer to shaping relationships between "things" (e.g. bodies, data, or ideas), positions the designer in relation to these "things" as objects of interaction, without precluding the designer being one of these objects. This design process is grounded in understandings of what these things are as objects of interaction, and therefore, what they can do, that are rooted in the conceptual frame and intentions of the designer. Many aspects of this conceptual frame may remain tacit and implicit throughout the design process-ideas about humans, non-humans, bodies, machines, technology, interaction, computation, space, time, gender, race, etc. Approaching interaction design by engaging in processes intended to make these aspects of the conceptual frame, and the values that underlie its construction, explicit, offers opportunities to treat these constitutive differences which bound conceptions of "things" as objects themselves in the design process.

We propose that interaction design be coupled with intra-active design, i.e. a continual and explicit engagement in the local production of subjectivity, which positions the interaction designer as an entity in the context of interaction. The concept of "intra-action" is not offered here as a replacement for, or redefinition of "interaction." Each of these concepts only becomes meaningful through its situated use and utility, which is defined, in part, by its continual differentiation from the other. By differentiating between interaction and intra-action we do not arrive at a binary explanation; rather, the concepts of interaction and intra-action only come to matter intra-actively in a given design practice. Interaction and intra-action become entangled in any examination of the ways in which the value systems of the designers have become inscribed within the technologies and techniques of an interactive system. For example:

Imagine that we wish to design a device like the Microsoft Kinect-an array of cameras and microphones with a microprocessor that produces an estimate of the number of human bodies in its field of vision and a representation of the positions of the joints of those bodies. During the design process the designers must answer, implicitly and/or explicitly, questions concerning what constitutes a human body and how it will be represented. Does it have four limbs? If not, how many can it have? Can it be in a wheelchair? A bed? What types of clothing can it be wearing? The designers also must ask where a body can be-can it be outside? Underwater? Is the body free to move through the same physical space as the device we are designing, or is it encumbered by the limits of the field of vision of the cameras? Answers to these questions, and no doubt a host of others, describe what bodies are and can do from the viewpoint of the object to be designed. As the object is built, these descriptions become inscribed in the hardware, software, operating instructions, training videos, etc., and ultimately produce a set of prescriptions for how, where, and what to be and do if one wishes to be identified as an object of interaction in this context. 
These prescriptions delimit the boundaries of a community populated by those who are able, willing, and interested to participate in interaction as put forward by the designers of a given technology. They are the foundations of culture, discipline, knowledge, and power in this community, and construct the group of objects available for interaction, as well as the locallyderived concept of interaction itself. In this paper, we shift our focus from the design of interaction between objects to the situated design of the culture organizing things that interact. This requires attention to those aspects of interaction design concerned with the construction of boundaries for the purposes of organizing objects of interest.

Cultures and their constituent parts-disciplines, knowledge, subcultures, politics, conflicts, etc.are not stable, and cannot be represented in their totality through any means. The process of referencing a community, a culture, a discipline, is a process of stabilization through various forms of in-, ex-, oc-, and transclusion. This process is the construction of a social object belonging to the community engaged in observation and reference; to say that this object is distinct from the community being observed would be to engage in a second processual construction of a community as a social object. Such an object, related to Durkheim's concept of a "social fact," is itself a process in relation with those engaged in its construction, and, as such, may not be referenced or represented in its totality. The relationship of these societies with those who construct them depends on the ways in which their borders have been designed to include, exclude, occlude, or transclude their designers.

Judith Butler points to the haunting of constructed borders by all that has been rendered invisible, yet remains $(1993,8)$. The absent presence of objects of non-interest is what stabilizes interpretations of objects of interest within a locally-derived system of interaction. That which constitutes a body in the eyes of the Kinect (or more generally, that which constitutes a body or object of interest in a system of classification), is set against that which it views as lacking bodily coherence, a view that overlaps with that of the designers. This body and other-than-body require one another in order to differentiate their ontologies through an ongoing process of constitutive constraint, which is never resolved once and for all. It warrants consideration then: "Given this understanding of construction as constitutive constraint, is it still possible to raise the critical question of how such constraints not only produce the domain of intelligible bodies, but produce as well a domain of unthinkable, abject, unlivable bodies?" (xi). Through their shared becoming, bodies of interest and bodies of non-interest remain entangled and intra-active, haunting one another as exteriorities within, resisting binary designation, and threatening the sovereignty of the local system of interaction. The "filtered-out" bodies, gestures, and "noise" of an interactive system remain integral to the coherence and operation of that system. Reiterating, with difference:

\footnotetext{
it is not enough to claim that human subjects are constructed, for the construction of the human is a differential operation that produces the more and the less "human," the inhuman, the humanly unthinkable. These excluded sites come to bound the "human" as its constitutive outside, and to haunt those boundaries as the persistent possibility of their disruption and rearticulation. 
When the sanctity of the objects of interest is undermined by the absent presence of their obligatory others, the designer must look beyond the discretized frame of interaction, towards the continual field of intra-action of which they themselves are part.

Bring your attention to the gradual changes in tempo of the heart you are holding. Speeding up as the lungs are filled, slowing down as the breath is released. Stay here for a moment, just focusing on the gradual shifts in tempo of the heartbeat with the breath.

Now bring your attention to the pulses themselves, those movements that fill your hands and produce tempo. Feel the ways each beat marks and divides the breath, and the ways the breath groups the beats. Perhaps those groups begin to take on character, shape, meter.

Take note of moments when a beat happens to occur at the moment of a transition from an inhale to an exhale, or an exhale to an inhale. Or a beat that happens to perfectly divide the breath into equal halves.

Now bring that same attention to the beats that fall in relations with the breath that are not so simple to categorize. Moments when the beats seem to be floating against the metrical regularity of the breath, before they lock back in briefly, only to float away again.

INTRA-ACTIVE DESIGN AS COLLECTIVE SUBJECTIVATION: FROM CAUSALITY TO ENTANGLEMENT

Intra-action is a neologism coined by Karen Barad which she describes as signifying "the mutual constitution of entangled agencies" and can be understood as shifting the focus from the individualistic notion of things interacting and cause/effect dualism to the material-discursive production of subjects and objects that intra-act $(2007,33)$. In her article "Quantum Entanglements and Hauntological Relations of Inheritance: Dis/continuities, SpaceTime Enfoldings, and Justice-toCome," she elaborates:

In contrast to the usual 'interaction', the notion of intra-action recognises that distinct entities, agencies, events do not precede, but rather emerge from/through their intra-action. 'Distinct' agencies are only distinct in a relational, not an absolute sense, that is, agencies are only distinct in relation to their mutual entanglement; they don't exist as individual elements. Importantly, intra-action constitutes a radical reworking of the traditional notion of causality. (Barad 2010, Note 1, 267, original italics)

While discourse about interaction, itself a thing, requires things that interact, discourse about intraaction involves processes of stabilization and destabilization (of these processes), the continual 
making and unmaking of things. Whereas things like interaction are often visualized using arrows to represent information moving from one object to another, an aspect of intra-action concerns the way in which those arrows and their directionality get constructed-the notion of cause and effect is replaced by entanglement.

Similar ideas can be found in the writings of Deleuze and Guattari:

\begin{abstract}
Mimicry is a very bad concept, since it relies on binary logic to describe phenomena of an entirely different nature. The crocodile does not reproduce a tree trunk, any more than the chameleon reproduces the colors of its surroundings. The Pink Panther imitates nothing, it reproduces nothing, it paints the world its color, pink on pink; this is its becoming-world, carried out in such a way that it becomes imperceptible itself [...] ([1980] 1987, 11)
\end{abstract}

It is not enough here to reverse the arrows and say that the tree trunk reproduces the skin of the crocodile-that is simply a judgment made from another perspective framed by mimicry, imitation, reproduction, evolution, etc. Another way to say this is that the chameleon is a process in continual change (becoming), and as such cannot be represented through any means, material, discursive, or otherwise. When we label it "the chameleon" and discuss what it may or may not be doing when it "changes color," we have produced a necessarily incomplete representation of it-a new objectthat omits an infinitude of aspects that fade into imperceptibility. What was omitted was done so due to our context, our frame of reference, and our current set of intentions concerning our construction and use of the chameleon (i.e., making a point in this paper).

Conceptual shifts such as these are radical as they are invitations to uproot the settled knowledge and resolved disputes of a discipline, to probe those aspects of disciplinary knowledge that ground practice. Just such a shift formed the basis of the approach to what Félix Guattari and Jean Oury referred to as "institutional psychotherapy" as practiced at the psychiatric clinic of La Borde since the 1950s where "everything there is set up so that psychotic patients live in a climate of activity and assume responsibility, not only with the goal of developing an ambience of communication, but also in order to create local centres for collective subjectivation. Thus it's not simply a matter of remodeling a patient's subjectivity-as it existed before a psychotic crisis—but of a production sui generis" (Guattari 1995, 6). This approach has profound implications not just for the working methods of the institution, but the institution itself: "one could not consider psychotherapeutic treatment for the seriously ill without taking the analysis of institutions into account. Reciprocally, the conception of individual treatment came to be revised, bringing greater attention to the institutional context" (Guattari 2015, 61). Guattari's views represent a turn away from individualism:

\footnotetext{
So we are proposing to decentre the question of the subject onto the question of subjectivity. Traditionally, the subject was conceived as the ultimate essence of individuation, as a pure, empty, prereflexive apprehension of the world, a nucleus of sensibility, of expressivity - the unifier of states of consciousness. With subjectivity, we place the emphasis instead on the founding instance of intentionality. This involves taking the relation between subject and object by the middle and foregrounding the expressive instance. (Guattari 1995, 22)
} 
In the context of interaction design, "taking the relation between subject and object by the middle" requires letting go of interpretations of mimicry and causality between pre-constituted "things." Only through their differentiation within processes of subjectivation and intra-action do discrete "things" become, and therefore become available as objects that can be made to interact.

Continuing to stay attentive to the complex temporal relationship between the breath and the heart, begin to imagine the hearts of the other two breathers in the room with you. Imagine that although their breathing is the same as the person in front of you, and their hearts follow similar patterns of acceleration and deceleration, the three of them produce a complex counterpoint of pulses out of which you find yourself in the continual process of making and unmaking rhythmic patterns, all against the cantus firmus of the breath.

\section{INTERLUDE: WHAT-WE-MEAN-BY-INTERACTION}

We pause here to bring awareness to our own process of weaving together appropriated passages of text by immanent philosophers in order to construct a narrative that is ours, not theirs, and designed to convince you the reader to consider our frame of reference. These authors and passages have been chosen strategically not just for the profundity of their ideas, but with full knowledge of our use of their discursive gravity as a technology of power. "My inhibitions, as you can see, can be expressed only by being dressed up in external statements, and now that I am using quotations as weapons of debate, I will offer some more in the hope of salvation" (Guattari 2015, 208):

Discourse is not what is said; it is that which constrains and enables what can be said. Discursive practices define what counts as meaningful statements. Statements are not the mere utterances of the originating consciousness of a unified subject; rather, statements and subjects emerge from a field of possibilities. This field of possibilities is not static or singular but rather is a dynamic and contingent multiplicity. (Barad 2003, 819)

Discourses are not once and for all subservient to power or raised up against it, any more than silences are. We must make allowance for the complex and unstable process whereby discourse can be both an instrument and an effect of power, but also a hindrance, a stumbling block, a point of resistance and a starting point for an opposing strategy. Discourse transmits and produces power; it reinforces it, but also undermines and exposes it, renders it fragile and makes it possible to thwart it. (Foucault [1976]1990, 100-101)

To what extent does discourse gain the authority to bring about what it names through citing the conventions of authority? And does a subject appear as the author of its discursive effects to the extent that the citational practice by which he/she is conditioned and mobilized remains unmarked? Indeed, could it be that the production of the subject as originator of his/her effects is precisely a consequence of this dissimulated citationality? (Butler 1993, xxi) 
Papers such as these are often described as contributions to this or that field; however, in consideration of the frame of reference established in the previous sections, we must acknowledge the inherently intra-active nature of such a contribution. We see this work as a contribution to the discourse intended to shape the contexts in which conceptions of "interaction" and "things that interact" are produced. The production of a definition of "interaction" is the production of subjects included within the boundaries of that definition, as well as the domain of the excluded, who come to haunt that boundary. The design of interaction necessarily includes becoming subject to "whatwe-mean-by-interaction."

Intra-action is related to collective modes of work such as collaboration, cooperation, coordination, teamwork, etc., but generalized to take into account that these notions themselves are produced through intra-action and that the participants, human or otherwise, are never stable, but are in continual processes of becoming and unbecoming. Intra-action can be seen as the dark matter that binds these continually changing, partially understood objects together in an ontogenetic becoming-universe.

The primary question with which we are concerned is how, and to what extent, we may design that universe, and what is at stake in the choice of different approaches. In the following sections, we investigate the concepts of boundary objects, diffraction, and critical appropriation as potential approaches to intra-active design.

Return your attention to the pulsing heart in your hands.

IS THIS A BOUNDARY OBJECT?

In the social sciences broadly, and human computer interaction and science and technology studies specifically, the concept of "boundary objects" is often deployed to describe and facilitate interaction between communities. Introduced by Susan Leigh Star in 1989, the concept was also intended as a provocation to the artificial intelligence community to reconsider notions of what constitutes intelligence from the standpoint of cooperative work in open systems. The following year, Star and James Griesemer refined the concept, demonstrating and advocating its use as an analytical tool to frame the cooperative actions of the players involved in the early years of UC Berkeley's Museum of Vertebrate Zoology (Star and Griesemer 1989). By way of a definition, Star and Griesemer offer the following:

Boundary objects are objects which are both plastic enough to adapt to local needs and the constraints of the several parties employing them, yet robust enough to maintain a common identity across sites. They are weakly structured in common use, and become strongly structured in individual-site use. These objects may be abstract or concrete. They have different meanings in different social worlds but their structure is common enough to more than one world to make them recognizable, a means of translation. (Star and Griesemer 1989, 393) 
Star and Griesemer write that the production and management of boundary objects "is a key process in developing and maintaining coherence across intersecting social worlds" (Ibid.). Isto Huvila goes a step further, stating that they "are a precondition for communication, cooperative work, and having reached mutual goals" $(2011,3)$. Star and Griesemer describe boundary objects as an expansion of what Callon called interessement: "Interessement is the group of actions by which an entity [...] attempts to impose and stabilize the identity of the other actors it defines through its problematization. Different devices are used to implement these actions" (Callon 1986, 8). While these two analytical approaches are similar, Star and Griesemer emphasize the construction and use of objects that get created to negotiate the boundaries between viewpoints rather than the processes of translation of information as it crosses boundaries.

For Star, the term "object" is meant to be understood in a variety of pragmatic, disciplinary, and material senses, as

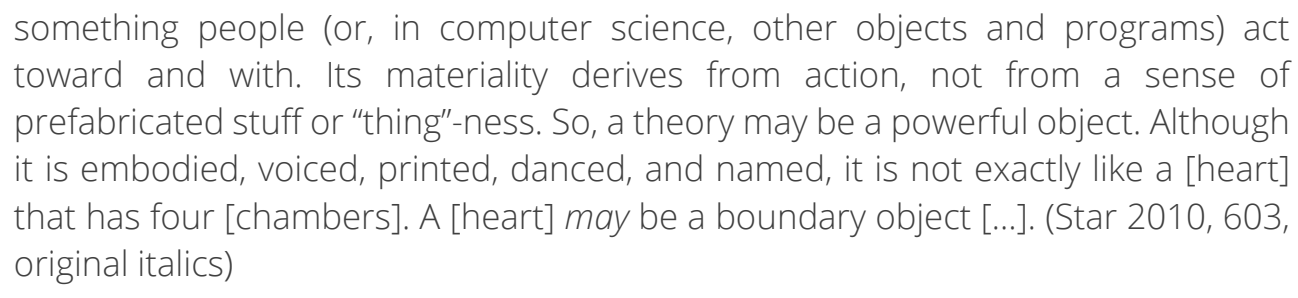

The point being made here is that the materiality of an object, be it a theory or a heart, does not determine whether or not it is or can be a boundary object. Rather, it is the differential materialization of this object through its performance within overlapping systems and worlds, always underway and always in relation to the materialization of other objects, that informs interpretations of it as a boundary object.

Imagine a healthy, beating heart, fully functional in its capacity as an organ sustaining life in a young pig. In this capacity, it sustains the basic biological systems of the body, but also requires those same systems in order to function. Now imagine the heart, i.e. the muscle, cut from those systems and removed from the pig. Displaced from its role in the sustenance of the body in which it was grown, it is now free to be used in other ways, as food, as an object in a piece of art, or perhaps even, as seems likely at the time of this writing, it may find use as a functioning heart again, ensuring the longevity of a being from another species. The specific heart that we are imagining was one that was bought from a triperie in Nice; it was intended to be sold for food, and indeed, we intended to eat it, but only after filming it as an object of choreography. We spent two days exploring and filming intimate touch with each other and the heart, after which, we could no longer imagine the heart in its capacity as food.

So, is this pig's heart a boundary object? This question requires the construction of a context in which this question matters. We could discuss its role in terms of capitalism, the geopolitics of meat consumption, animal rights. We could also discuss it in terms of its role in shaping the exploration of touch and movement in an artistic context between two people with different disciplinary backgrounds. We can also see the construction of the pig's heart through description 
and recollection for the purpose of arguing that boundary objects are always constructed $a$ posteriori in order to shape the viewpoint of a peer group, rather than through use as suggested by the literature. There is a grave risk for us here that one day you may encounter the short film in which we handle the pig's heart and see it as an example used to make a claim about boundary objects, or worse, you may think that it was produced with that purpose in mind (we assure you it was not).

The point being made here illustrates a bifurcation of a boundary object into an object constructed through cooperative work, and an analytical object used to describe said work. An ethnographer studying cooperative work is also implicated in cooperative work. For the people under study, theories and other "things" have the potential to become boundary objects in the context of their cooperative work. However, when described as such by the ethnographer, these objects, along with the people that use them, may also become boundary objects in the production of knowledge between the ethnographer and the ethnographer's peer group. This is to say that the construction of a boundary object to describe aspects of collective work becomes a boundary object in the collective work practice of description. In Callon's study of the different parties involved in the preservation of sea scallops in St. Brieuc Bay, he astutely implicates the disciplinary peer group that the Japanese researchers are affiliated with as contributing to the intentions of the researchers (Callon 1986). Similarly, in order to situate this discussion of boundary objects in a larger discourse, we must implicate ourselves as part of the peer group associated with Star, Griesemer, and those others who contribute to the production of knowledge related to boundary objects.

In this very text, we appropriate the concept of boundary objects-which can itself be used as a boundary object-and employ it in order to question the capacity of boundary objects to support approaches to interdisciplinary collaboration that not only tolerate divergent viewpoints, but that require divergent viewpoints to operate and flourish. As a potential boundary object, this paper is part of a context of intra-action that includes other publications about boundary objects that are themselves potential boundary objects.

Bring as much of the palms of your hands and your fingers into contact with the surface of the heart as you can. Try to envelope it fully so that you can feel as much of the subtle complexity of its movement as possible. Let the discreteness of 'beats' dissolve into continuous and continual waves of pulses. Feel them travel across, through, between your hands. Try to imagine that in the smallest movements, the ones barely perceptible, the heart is gathering the energy it needs to beat. Now imagine that you can increase that energy by squeezing it in those moments between the beats, pushing energy into the heart, and receiving it back in your hands when it beats. 


\title{
HAUNTED BOUNDARIES [OF BOUNDARY OBJECTS]
}

Star and Griesemer assert the "fundamental sociological finding" $(1989,388)$ that consensus is not necessary for cooperation; however, their text belies the belief that some form of reconciliation of differing viewpoints is necessary for cooperation:

Consensus is not necessary for cooperation nor for the successful conduct of work. [. . .] However, scientific actors themselves face many problems in trying to ensure integrity of information in the presence of such diversity. [...] When the worlds of these actors intersect a difficulty appears. The creation of new scientific knowledge depends on communication as well as on creating new findings. But because these new objects and methods mean different things in different worlds, actors are faced with the task of reconciling these meanings if they wish to cooperate. This reconciliation requires substantial labour on everyone's part. Scientists and other actors contributing to science translate, negotiate, debate, triangulate and simplify in order to work together. (Star and Griesemer 1989, 388-389)

The characterization that diversity causes problems, that the intersection of worlds creates difficulty, situates the concept of boundary objects as the key to reconciling these meanings so that communication can occur and new scientific knowledge can be created. Boundary objects are positioned as passage points through which information must flow for cooperative work to produce knowledge. What a reconciliation of viewpoints implies, and the ways it differs from consensus, has been examined by Huvila who writes that, "the creation or reshaping of a boundary object is always an attempt to make a hegemonic intervention" $(2011,21)$. He elaborates:

\begin{abstract}
the boundary object and boundary crossing have a purpose. This purpose, even a very lenient one, is an attempt to influence adjacent communities and as such a more or less belligerent form of hegemonic intervention. [...] A specific characteristic of a boundary object is that it makes hegemonic interventions easier to accept for communities with antagonistic tendencies even if the emerging norm would be advantageous by default only from the point of view of the hegemonic position. Boundary objects may thus be seen as facilitators of hegemonic interventions of different levels embedded in the boundary practices of interfacing communities. (Huvila 2011, 21)
\end{abstract}

The construction of this hegemonic relationship is the construction of a particular type of interaction, which itself occurs in the context of intra-action and the production of truth/power. While the subjugation or assimilation of viewpoints as they funnel through the passage point of the boundary object may be consensual or even welcome, the dynamics of boundary crossing cannot be interpreted outside of the operation of power. In Foucault's estimation:

In itself the exercise of power is not violence; nor is it a consent which, implicitly, is renewable. It is a total structure of actions brought to bear upon possible actions; it incites, it induces, it seduces, it makes easier or more difficult; in the extreme, it constrains or forbids absolutely; it is nevertheless always a way of acting upon an 
acting subject or acting subjects by virtue of their acting or being capable of action.

A set of actions upon other actions. (1982, 789)

What Huvila is effectively proposing is a category of boundary objects that we might call "hegemonic boundary objects" that are used to conduct and describe collective work that is based in particular assumptions about productivity rooted in Capitalism. The production of these hegemonic boundary objects in analytical discourse reflects the analyst's frame of reference, intraactive context, and operations of power. Actions are not themselves a priori hegemonic in nature; the determination that an action is or was hegemonic is a situated one that requires the production of a subjectivity in which the action becomes hegemonic.

While our purpose here is to highlight the subjective nature of such a determination in order to invite discourse about other possible subjectivities, doing so pushes us into delicate ethical territory where questions of accountability and responsibility must be raised, both with respect to the act deemed hegemonic, and the act of deeming an act hegemonic. The proposition that these determinations are situated in one's subjectivity does not imply absolution of responsibility; on the contrary, their production is entangled with questions of responsibility. Responsibility requires things to be responsible to, and the production of those things cannot be distinguished from acts of responsibility.

In a similar vein to Huvila's account of boundary objects as hegemonic interventions, Kathryn Henderson describes "conscription devices" as visual representations used to enlist and organize participation in collective work. She states: "Since visual representations are located at the center of power, they are the locus of action, which may be negotiation and consensus, or it may be conflict and power plays" $(1999,134)$. This notion of power, limited in its conceptual and geographic reach by its ocularcentrism, is very different than Foucault's "total structure of actions brought to bear upon possible actions" (1982, 789). Power cannot be reduced to control. The characterization of power as a central hub (i.e. a conscription device), that mediates between the interests of parties during interaction, is a colonialist narrative: it fails to acknowledge the continual, intra-active construction of the haunted boundaries of these objects, as well as the infrastructures they traverse.

Elaborating on the concept of boundary objects, Bowker and Star discuss "boundary infrastructures" as "objects that cross larger levels of scale than boundary objects" to "deal with regimes and networks of boundary objects (and not of unitary, well-defined objects)" (Bowker and Star 1999, 313). They explain that, "[w]hat we gain with the concept of boundary infrastructure over the more traditional unitary vision of infrastructures is the explicit recognition of the differing constitution of information objects within the diverse communities of practice that share a given infrastructure" (314). While "scaling up" from objects to infrastructure (another type of object, in our estimation) grants perspective on the systemic operations of power, the plasticity and translatability of a boundary infrastructure between communities still requires the management of "divergent viewpoints" by way of "accommodations, work-arounds, and in some sense, a higherlevel of artful integration" (292). 
To this end, we inquire: just how little overlap, or conversely, just how much difference can a boundary object, conscription device, or boundary infrastructure tolerate before one's notion of cooperation or collaboration breaks down?

In her 2007 article "Boundary Negotiating Artifacts: Unbinding the Routine of Boundary Objects and Embracing Chaos in Collaborative Work," Charlotte Lee provides a substantial critique of the community's adoption and use of boundary objects. In her estimation, the problem lies not in the conception of boundary objects themselves, but rather in the "tendency of researchers to label every artifact [that moves between communities of practice] a boundary object" because "it forces us to deny what we observe, to ignore the finer points of the boundary object definition, or to awkwardly wrap new theories around the [definition of boundary objects]" $(2007,314)$. Lee points out that in their original conception, "ultimately boundary objects was posited as a creature based on established standards" and that "[t]he dependence of boundary objects on the premise of established standards is inherently problematic for theorizing incipient, non-routine, and novel collaborations" (Ibid.). To account for artifacts that exist in "projects that are fairly non-routine and fairly complex," Lee introduces the term "boundary negotiating artifacts" (334). What is at stake in her critique of her community's (over)use of boundary objects is the neglect of work practices that do not appear similar to those that gave rise to boundary objects in the first place. Or worse, perhaps, that the study of "incipient, non-routine, and novel collaborations" will be misconstrued, a risk that puts her community's knowledge on shaky foundations.

In her conclusion, Lee questions whether the focus on "standardized artifacts and stable organizational contexts" is due to them being "most easily codified into our computational systems" (336). While this is surely not the only reason, it highlights the role of the observer, the person ultimately responsible for the construction of boundary objects / boundary negotiating artifacts. A description of a boundary object or a boundary negotiating artifact is an expression of the frame of reference from which the observer views the world, an expression that is then performed by the reader in the context of the discourse that constitutes the practice of boundary object production.

In the framing of boundary objects, boundary negotiating artifacts, and boundary infrastructures as a means for cooperation and reconciliation-in service of a shared goal or greater good-the non-neutrality of the boundary as common territory must be taken into account. Designating a border territory or "common ground" for passage and translation between communities requires the drawing of a boundary around this shared space that may not be symmetrical in its inclusivity and reflectivity of interests. When cooperation, reconciliation and collaboration are promoted under the guise of accessibility and diversity, it is important to shed light on colonial and capitalist imperatives to standardize methods and maximize productivity through the self-regulatory and disciplinary effects of power.

While the concept of boundary objects (and its variants) tolerates some degree of difference, it cannot accommodate irreconcilable difference in which there is no reflection of viewpoints between communities. By requiring mirrored interests as the basis for plasticity and 
translatability - and therefore visibility - the framework of boundary objects positions difference in opposition to similarity, and dissent against consensus.

In time with the person whose heart pulses in your hands, gradually begin to accelerate the pace of your breathing, fully emptying and filling your lungs with each breath. In through your nose, out through your mouth. As you continue to speed up, taking in much more oxygen than your body needs, you may find that the air feels cold in your nose, in your throat, in your chest. Continue faster still. Your

fingers may begin to tighten and curl, locking on to the heart. This is normal, it will pass. Faster still. Feel the heart in your hands beat faster and more regularly as both of you continue to accelerate. Inhale, exhale, inhale, exhale, inhale.

\section{INTERLUDE: A FAILED ATTEMPT AT REFLEXIVITY}

Here's the thing. Boundary objects are frightening for us because they represent what we perceive to be a practice of colonizing knowledge by recharacterizing it in order to serve the needs of a different community. What we have to account for now is the way in which we performed exactly the same act in order to make our point about it.

Our intent as authors, if such a thing can be trusted, is not to interact with you, the reader, through the transmission and translation of ideas towards shared understanding. We simply do not believe that such authority exists. And yet, our authorial attempts to reflexively account for our role in this discourse are not sufficient to reveal our own sense of intentionality, for in reflexively reflecting ourselves back to ourselves, we cannot but see our own vision of ourselves.

\section{[R]eflexivity is nothing more than iterative mimesis: even in its attempts to put the investigative subject back into the picture, reflexivity does nothing more than mirror mirroring. Representation raised to the nth power does not disrupt the geometry that holds object and subject at a distance as the very condition for knowledge's possibility. Mirrors upon mirrors, reflexivity entails the same old geometrical optics of reflections. (Barad 2007, 87)}

The problem is that a reflexive accounting of ourselves as authors in the story we are trying to tell never really brings new information to that story, it simply tells a different story, one that includes us, an accounting for which we must account, reflexively, on and on.

What we must do is bring into question the intra-actively enacted boundaries of ourselves as selves with the authorial capacity to produce an object such as this text, or a shared goal such as this: As authors we seek to articulate approaches to design, in and between multiple communities of practice, that not only tolerate difference, but rather, that require critical difference between practices in order to thrive. 
Our use the term critical here and elsewhere is an amalgam of its definitions and stands in for the assertion that the noun it describes matters in a given context. It is not enough to speak of difference, but rather difference that has crossed an inflection point, difference that has been brought to a point of critical mass, difference that is essential to a context and that must be cared for in order to prevent it from becoming flattened.

In caring for the critical differences that emerge in our designing of interaction as composerchoreographer, we desire strategies that are not based on critique, for as Barad reminds us:

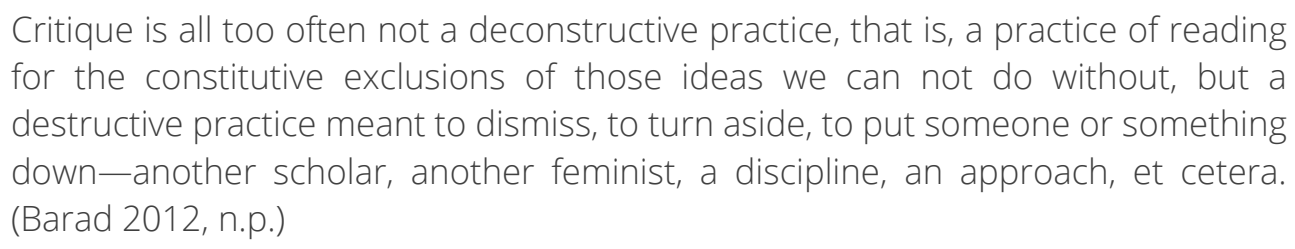

Our critique in the preceding sections is not a critique of the concept of boundary objects, rather it is a critique of the act of constructing boundary objects. Such an act involves the construction of a community, a practice, and a discourse through the making of their boundaries, and what we have tried to show is that the making of those boundaries is the expression of value systems. The problem is that in order to express our critique of these kinds of acts, we first had to construct a community, a practice, and a discourse in which these acts were performed. It's not enough to say that we did this-to admit our culpability-nor is it enough to point out that the act of critique is an act of making communities that can be critiqued. When communities are made, they are always in relation to the things they are not, including the continual acts of their making, and our goal here is to foreground, so that we may better care for, the relationships that ultimately form these communities.

In search of approaches to designing interaction that bring awareness to the value systems involved in the local production of "interaction" and "things that interact," we appropriate Barad's appropriation of "diffraction" (from quantum physics into agential realism), as a method to examine "patterns of difference that make a difference" between communities of research. Further, we discuss the potential value of what we term "critical appropriation," i.e. the recontextualization of technologies across disciplinary boundaries, with care for perceived transgressions of the ethical norms that govern notions of proper use and mastery in the respective domains. Our use of the term "appropriation" is itself an appropriation and repurposing of this term to resist, rather than facilitate, the colonizing of knowledge by recharacterizing it in order to serve our own needs. Following the logic developed so far in this text, our interpretation of the concepts of diffraction and critical appropriation can only be understood through ongoing intra-action with concepts introduced earlier in the text, such as interaction, reflexivity, and boundary objects.

Hold your breath. 


\title{
DIFFRACTION: CRITICAL DIFFERENCE AND RESPONSIBILITY IN COLLABORATION
}

Barad builds on the metaphor of "diffraction," proposed by Haraway (1992), as a strategy to resist framing the "geometrical optics of reflection" as the source of difference between phenomena (Barad 2007, 87). Whereas reflection involves the bouncing back of a wave (e.g. of a light, water, or sound wave) from a surface, diffraction is the changing of direction of this wave as it passes around the edges of an obstacle or through a narrow opening. As a wave in the ocean encounters a rock that breaks the surface, the wave bends around the rock and interferes with itself on the other side. The effects of this interference are as much the result of the characteristics of the wave as they are of the size and shape of the rock. Unlike mirrors which produce images of objects placed at a distance, "diffraction gratings are instruments that produce patterns that mark differences in the relative characters (i.e., amplitude and phase) of individual waves as they combine" (81). In appropriating the concept of diffraction in order to investigate how different differences come to matter, Barad highlights that:

\begin{abstract}
[diffraction] is not just a matter of interference, but of entanglement [...] There is not this knowing from a distance. Instead of there being a separation of subject and object, there is an entanglement of subject and object [...] instead of being about offering an undistorted mirror image of the world, it is about accountability to marks on bodies, and responsibility to the entanglements of which we are a part. (Barad 2012, n.p.)
\end{abstract}

This warrants emphasis: by deconstructing notions of reflexivity, individuals and institutions are not absolved of responsibility for the ways in which they are entangled with "others," for these others, to repeat from Butler, are what come to bound their "constitutive outside, and to haunt those boundaries as the persistent possibility of their disruption and rearticulation" $(1993,8)$. Subverting hegemonic narratives of control-based interaction between subjects, the intra-active character of
diffraction patterns illuminat[e] the indefinite nature of boundaries-displaying shadows in "light" regions and bright spots in "dark" regions-the relationship of the cultural and the natural is a relation of "exteriority within." This is not a static relationality but a doing - the enactment of boundaries - that always entails constitutive exclusions and therefore requisite questions of accountability. (Barad 2007, 135)

The enactment of boundaries does not necessarily, or perhaps ever, distribute territories and agencies symmetrically. As such, it is reductive (and potentially dangerous) to characterize power relations within and across these borders as a matter of interaction, that is, as reciprocal actions, effects, and influences. Framing interaction as reciprocal exchanges or mappings between things (people, objects, data, disciplines, cultures, etc.), does not account for shifting asymmetries between these things; it lays the foundation for subjugation by way of self-regulatory and disciplinary effects of power, and fails to implicate all participants in the "entanglements of which 
[they] are part" (Barad 2012, n.p.). An important distinction between reflection and diffraction is that:

\begin{abstract}
diffraction does not fix what is the object and what is the subject in advance, and so, unlike methods of reading one text or set of ideas against another where one set serves as a fixed frame of reference [i.e. interaction], diffraction involves reading insights through one another [i.e. intra-action] in ways that help illuminate differences as they emerge: how different differences get made, what gets excluded, and how those exclusions matter. (Barad 2007, 30)
\end{abstract}

Designing interaction involves the mapping of perceived difference between objects of interaction (e.g. bodies, gestures, physiological processes, media, etc.), within the frame of reference of the designers. What constitutes "difference that matters" within this locally derived system of interaction depends on the vision of the designers, which necessarily excludes types of difference that are invisible to them. Shifting focus from the reflective tactics of interaction design towards the diffractive performance of intra-action does not necessarily produce greater visibility of difference itself, for difference's sake. Haraway makes the important distinction that, "a diffraction pattern does not map where differences appear, but rather maps where the effects of differences appear" (Haraway 1992, 299, original italics). Mapping the effects of differences does not presume that these differences emerged from a unitary source. This is important, because it subverts the assumption that difference can only come to matter in opposition to an originary sameness. In this regard, Barad argues that:

\title{
If diffraction is to serve as an important metaphor for differences that matter, it is crucial that we pay attention to the kinds of differences that different understandings of diffraction evoke, so as to not conflate questions of accountability to differences that matter with postmodern celebrations of difference for difference's sake. (Barad 2007, 214)
}

In Barad's use of the term, diffraction stands in for at least three things: a theoretical description of the behavior of light and matter as both waves and particles; an ethnography of the development of quantum mechanics in response to a phenomenon that defied explanation in terms of classical physics; and a philosophical proposition that subject and object are not separate or separable. We are inspired by what we read in Barad's development of diffraction as a metaphor, but we must also acknowledge that we do not have the same relationship to science and philosophy that she does. Our background as choreographer-composer in no way precludes us from engaging in scientific and philosophical discourse, but we do have a different frame of reference and we are aware that the boundaries of diffraction, or rather, "what we mean by diffraction," are differently haunted for us.

Our appropriation of diffraction, and likewise boundary objects, is done to explore approaches to collective work, specifically different conceptions of interaction design such as composition and choreography, rooted in difference qua difference, rather than difference as defined with respect to sameness. It is an act no different than Barad's appropriation of Niels Bohr's formulation of 
quantum mechanics or Star and Griesemer's appropriation of the founding of the Berkeley Museum of Vertebrate Zoology, with the possible exception that we acknowledge the aporetic act of constructing a subject in order to argue that there is no a priori distinction between subject and object. This is to say, this presentation of diffraction and boundary objects is based in constructions of them-constructions that we have argued throughout the entirety of the paper are shaped as much by what they include as what they exclude.

For us, this is an exercise of critical appropriation, which we describe as a sustained act of appropriation that is continually destabilized by the provocation that things only become things through exclusion, that what is excluded forms the basis for other onto-epistemologies, and that we bear responsibility for that which we exclude. Paraphrasing Derrida, we are "responsible to anyone (that is to say, to any other) only by failing in [our] responsibility to all the others, to the ethical or political generality. And [we] can never justify this sacrifice; [we] must always hold [our] peace about it [...] What binds [us] to this one or that one, remains finally unjustifiable" $(2008,70)$.

As an approach to intra-active design, what the metaphor of diffraction offers, especially to those involved in interdisciplinary collaboration, is the proposition that it is possible to work togetherresponsibly-not only based on a mutual goal or common ground that reflects an overlap in viewpoints, but rather, through an investigation of how and why differences have come to matter within and between individuals, disciplines, and cultures over time. Approaching collective work diffractively requires awareness of the ongoing production of difference, not only quantitatively, but æsthetically and ethically, as it transforms with and through the entangled agencies of participants.

Having discussed the potential of boundary objects and diffraction as approaches to intra-active design by interaction designers across disciplines, we now discuss our conceptualisation and practice of interdisciplinary design by way of critical appropriation.

Resist the urge to release. You may feel your chest move involuntarily. This is your diaphragm spasming, trying to pull air into your lungs. Try to relax, and it will pass. Keep holding.

\section{CRITICAL APPROPRIATION: INNOVATION BY DESTABILIZATION}

Critical appropriation involves the continual de- and re-contextualization of technologies (concepts, tools, techniques, etc.) between disciplinary communities, with attention to shifts in ecology and epistemology, as well as perceived ethical transgressions (Naccarato and MacCallum 2017). Whereas appropriation may be associated with the reflective tactics of interactiondisplacing objects from one context to another without investigating how and why differences emerge-critical appropriation is necessarily diffractive: shifts in ecology and epistemology are positioned as objects of interaction in relation to appropriated materials. As a means to address non-consensus and conflict in interdisciplinary collaboration, critical appropriation differs from 
boundary objects in that it is not intended to mitigate divergence by translating differences into shared understanding, goals, and productivity. Critical appropriation is not a matter of adaptation in which objects "are weakly structured in common use, and become strongly structured in individual-site use," or in which "their structure is common enough to more than one world to make them recognizable, a means of translation" $(1989,393)$. Critical appropriation is a provocation to continue to understand objects (people, bodies, movements, gestures, concepts, disciplines, cultures, communities, infrastructures, boundaries, etc.) as inherently multiple, and to bring attention to their ontologies outside of their current subjectivity. This is not a matter of bending the ontologies of objects to fit them inside an ethico-æsthetic frame that is accessible to all parties in a project; rather, critical appropriation is entangled with "a politics and ethics of singularity, breaking with consensus" towards collective subjectivation (Guattari 1995, 117).

Importantly, a diffractive reading of boundary objects, diffraction, and critical appropriation, as well as interaction and intra-action, works to "illuminat[e] the indefinite nature of [their] boundariesdisplaying shadows in 'light' regions and bright spots in 'dark' regions" as well as their "constitutive exclusions and therefore requisite questions of accountability" (Barad 2007, 135). Each proposition addresses difference, power, and collaboration from a particular orientation within an entanglement of value systems, the effects of which are in continual intra-action through our appropriation and treatment of them in this paper.

In our collaborative practice as a choreographer-composer we place emphasis on critical appropriation of biosensors in creation and performance as a means to intervene in the invisible boundaries of our own ethico-æsthetic frame(s) of reference (Naccarato and MacCallum 2017). We often use electrocardiograms (ECGs) in a variety of contexts in our work: they are worn by dancers, their amplified and processed signals are listened to by musicians, and those same signals are felt by guests invited into the work. The critical appropriation of the ECG devices, the WiFi technology used to transmit their signals, and the hardware and software used to amplify, process, and transduce those signals so that they may be heard and felt, involves a sustained act of care for discourse surrounding their ontology, epistemology, ethics, and æsthetics. In this practice of critical appropriation, questions about what an ECG is and how it works are inseparable from questions about what the heart is and why we might want to measure it. Questions about how to relate to a sonified ECG signal in a musical context are inseparable from questions about how we conceive of musical time, and other temporalities. Practical questions concerned with how to properly wear an ECG and the effects of movement on the signal are inseparable from questions of physiological control, the relationship of space to time in dance, and the ways in which space and time are constructed. The goal here is to gain insight into our practices as they are disrupted at the point of their intersection with an object of incommensurate difference.

There is always risk that the transposition of specialized technology across disciplinary lines will compromise the rigour and scepticism of the respective disciplines. For example, in the appropriation of biosensors from medicine to artistic practice, diagnostic practice may be diminished, because artists have different disciplinary training, and divergent (non-medical) goals. Likewise, the rigour of compositional and choreographic practices may be undermined by the 
imitation of foreign techniques for research, which serve the intentions of an alternate discipline. Importantly, an artist employing a biosensor in a manner contrary to the training of a medical professional should not be seen as lacking that training any more than a nurse who uses a biosensor without considering the æsthetic implications of their actions should be seen as lacking artistic integrity. In scenarios of appropriation, the question is not whether the encountered value systems are good or bad or right or wrong, but rather, what goals these values propagate in a given disciplinarily-constituted, and therefore exclusive, context.

For example, consider the heart that continues to beat in your hands. It is incommensurable with the trace of an ECG. The ECG measures an aspect of the heart that we do not feel when we hold it in our hands. They are related and in relation, to be sure, but the ECG is not "the-thing-we-call-theheart," i.e. the seat of the soul, the originary source of music and love. The sensation of your heart pulsing in my hands is of an order of complexity that defies description in terms of dimensionality, space, and time. These beats that I feel are simultaneously neither-discrete-nor-continuous-yetboth-discrete-and-continuous (Maffie 2004, n.p.). They travel through and between both of my hands, which, as I slowly move them, continue to shift the origins and trajectories of discretocontinuous pulses. I placed my hands inside your chest because I wanted to be closer to you, to experience your heart directly, free from the noise of mediated experience. I wanted to hear, feel, see, and smell your heart, not your chest, your lungs, your skin, your electrical potential. But I am still on the outside, around your heart. I don't think I wanted to be inside your heart; I think I wanted to be coextensive with it.

Noise is a problem with all acts of measurement, but here, perhaps, "noise" can offer opportunities to explore what Guattari might call the "mutant coordinates" of a discipline. The pill that we are offering here is a hard one to swallow: we are suggesting that noise, the very "thing" that obfuscates the "thing" we are interested in, should be considered as an opportunity to question the foundations of our interest in that particular "thing"; that the noise that prevents us from recognizing a particular "thing" as an object of interest for interaction (be it a physiological process, a gesture, or a body) might be an indication that our notions of the constitutive boundaries of this "thing," are, in fact, noisy.

By paying attention to the noise of our own perception, which may be based on our disciplinary training and cultural context, as well as our material bodies and material practices, or our mood on a given day, we become implicated as subjects in the perceptual process. Likewise, by paying attention to the noise produced by the hardware and software, its designers and users, and the intra-active context of interaction, the exclusion of data from a signal, becomes evident. Importantly, there is no un-noisy or un-mediated signal, nor is there an un-noisy or un-mediated perception of this noisy signal. Noise is always present, and in cases of appropriation of technologies, interrogating so-called noise can point towards the operation of ethics within and between collaborators and disciplines.

What is at stake here is the construction of a discipline that no longer recognizes that there are conceptions of gestures, bodies, processes, or concepts, outside of its frame. Once we as a 
community have decided, implicitly or explicitly, what, for example, a gesture is, we can stop looking for gestures that fall outside of that spatio-temporal frame. The reorientation of the concept of gesture from descriptive to prescriptive brings with it an ethical weight that can stagnate a discipline through the subjugation of æsthetic exploration. That bears repeating: the moment that we make the transition from using the concept of gesture to describe aspects of a mover's movement, to prescribing that movement as a gesture, we have entered into ethical territory that begins to restrict æsthetic choice. This process of restriction is inescapable; our goal here is not to advocate for its dissolution, but rather for a continual process of disruption of ethical restrictions through critical appropriation, disciplinary collaboration and æsthetic exploration. Through the intersection of divergent value systems in disciplinary crossings, there is potential for disruption of the calcified conventions of each discipline-by all that haunts them-and therefore a reconfiguration of what is being included and excluded from their respective territories.

Guattari likens this type of destabilization to mutation and sees the reframing of disciplinary structures such as psychoanalysis in terms of ethico-æsthetics, rather than simply ethics, as key to resisting stagnation and homogenization.

\begin{abstract}
If we turn for a moment to a discipline like psychoanalysis, which claimed to affirm itself as scientific, it is increasingly clear that it has everything to gain from putting itself under the ægis of this new type of æsthetic processual paradigm. Only in this way can it reacquire the creativity of its wild years at the turn of the century. (Guattari 1995, 106)
\end{abstract}

In the field of psychoanalysis, this has deep implications that require no less than the redefinition of the body itself.

Let us take as a final example an open redefinition of the body, so necessary for
the promotion of therapeutic assemblages of psychosis: the body conceived as
intersection of partial autopoietic components, with multiple and changing
configurations, working collectively as well as individually; all "the bodies"--the
specular body, the fantasmatic body, the neurological corporeal schema, the
biological and organic soma, the immune self, the personological identity within
familial and environmental eco-systems, collective faciality, refrains (mythical,
religious, ideological...). (Guattari 1995, 117-118)

This passage is remarkable in its articulation of an aspect of psychotherapy, a predefined conception of the body and its binary relationship to the brain, that for many practitioners has been rendered invisible by the boundaries of their disciplinary training. Further, it points to the difficulty of the type of work we propose in this paper, which is to say, we are advocating for engagements with technology that are harder, not easier, slower, not faster, and which not only defy measurement and evaluation, but call into question the nature of their roles.

Our formulation of critical appropriation thus far has been with respect to the use of technology for purposes other than those for which it was designed. Critical appropriation, however, is a general, diffractive practice intended to bring awareness to, and care for, the multiplicity of an 
object and to prevent its collapse down to something perceived as singular by the practitioners operating inside the structure of an emerging discipline. We appropriate technologies critically as an intervention intended to destabilize the boundaries of our practice, but it is normal for new practices to emerge out of those interventions. As practices stabilize, they become technologies in the world, belonging to others than ourselves, until ultimately that which we engendered, we now appropriate, critically.

Critical appropriation is a destabilizing practice-a process of intervening in moments of relative comfort in order to interrogate aspects of practice that have stabilized and receded into the shadows. From the shadows, these elements continue to calcify into increasingly solid boundaries making voices that come from outside their borders increasingly less intelligible. These boundaries form the ethics of a discipline-they separate those actions that cannot be done from those that must. They produce language that renders utterances unintelligible and construct the machinery that allows actions to be seen as transgressions. To repeat more generally, what is at stake here is the construction of disciplines that can no longer recognize forms of knowledge and know-how that operate outside of, yet continually haunt their boundaries.

Release the air in your lungs as you gently release the heart in your hands. Bring awareness to the rising and falling of your pulse as you breathe deeply and slowly, in through the nose, out through the mouth. Find a position that's comfortable as you recover your breath.

\section{POSTLUDE: APOLOGIA}

Wherever you are, imagine you are us, and there are three people sharing the space with you: Karen Barad, Michel Foucault, and Félix Guattari. They are seated or standing, silent, relaxed, simply listening and observing. You, choreographer-composer, in making a point to yourself, composer-choreographer, reach for a text you did not write, wielding it as a weapon of debate, subject to the gaze of the person who wrote it and their peers.

Appropriation is always violent, and we have engaged in it throughout this paper; for that we apologize, especially to those from whom we have taken and mis-re-presented. Appropriation is not ok and cannot be made ok by acknowledging or apologizing; the only thing we can do is act responsibly inside of the context of our own making. The words in this paper are ours, even those we attribute to other authors-and now they are yours. 


\section{Works Cited}

Barad, Karen. 2014. "Diffracting Diffraction: Cutting Together-Apart." Parallax 20 (3): 168-187. https://doi.org/10.1080/13534645.2014.927623

. 2012. "Matter Feels, Converses, Suffers, Desires, Yearns and Remembers." In New Materialism: Interviews \& Cartographies. Edited by R. Dolphin and I. van der Tuin, n.p.. Michigan: Open Humanities Press.

2010. "Quantum Entanglements and Hauntological Relations of Inheritance: Dis/continuities, SpaceTime Enfoldings, and Justice-to-Come." Derrida Today 3 (2): 240-268. https://doi.org/10.3366/drt.2010.0206

__- 2007. Meeting the Universe Halfway: Quantum Physics and the Entanglement of Matter and Meaning. Durham, NC: Duke University Press. https://doi.org/10.1215/9780822388128

___. 2003. "Posthumanist Performativity: Toward an Understanding of How Matter Comes to Matter." Signs 28 (3): 801-831. https://doi.org/10.1086/345321

Bowker, Geoffrey C., and Susan Leigh Star. 1999. Sorting Things Out: Classification and Its Consequences. Cambridge, MA: MIT Press.

Butler, Judith. 1993. Bodies That Matter: On the Discursive Limits of "Sex." New York: Routledge.

Callon, Michel. 1986. "Some Elements of a Sociology of Translation: Domestication of the Scallops and the Fishermen of St Brieuc Bay." In Power, Action and Belief: A New Sociology of Knowledge? Edited by John Law, 196-233. London: Routledge.

Deleuze, Gilles, and Félix Guattari. (1980) 1987. A Thousand Plateaus: Capitalism and Schizophrenia. Edited and Translated by Brian Massumi. Minneapolis: University of Minnesota Press.

Derrida, Jacques. 2008. The Gift of Death. Edited and Translated by David Wills. Chicago: University of Chicago Press. Foucault, Michel. (1976) 1990. The History of Sexuality: Volume 1: An Introduction. New York: Random House. 1982. "The Subject and Power." Critical Enquiry 8 (4): 777-795. https://doi.org/10.1086/448181

Guattari, Félix. 1995. Chaosmosis: An Ethico-Aesthetic Paradigm. Edited and Translated by Paul Bains and Julian Pefanis. Indianapolis: Indiana University Press.

2015. Psychoanalysis and Transversality. South Pasadena: Semiotext(e).

Haraway, Donna. 1991. "A Cyborg Manifesto: Science, Technology, and Socialist-Feminism in the Late Twentieth Century." In Simians, Cyborgs and Women: The Reinvention of Nature, 149-181. New York: Routledge.

___. 1992. "The Promises of Monsters: A Regenerative Politics for Inappropriate/d Others." In Cultural Studies. Edited by Lawrence Grossberg, Cary Nelson, and Paula A. Treichler, 295-339. New York: Routledge.

Henderson, Kathryn. 1999. On Line and On Paper: Visual Representations, Visual Culture, and Computer Graphics in Design Engineering. Cambridge, MA: MIT Press.

Huvila, Isto. 2011. "The Politics of Boundary Objects Hegemonic Interventions and the Making of a Document." JASIST 62 (12): 387-406. https://doi.org/10.1002/asi.21639

Lee, Charlotte P. 2007. "Boundary Negotiating Artifacts: Unbinding the Routine of Boundary Objects and Embracing Chaos in Collaborative Work." Computer Supported Collaborative Work 16: 307-339.

Maffie, James. 2014. Aztec Philosophy: Understanding a World in Motion. Boulder: University Press of Colorado.

Star, Susan Leigh. 1989. "The Structure of Ill-Structured Solutions: Boundary Objects and Heterogenous Distributed Problem Solving." Distributed Artificial Intelligence 2: n.p.

___. 2010. "This Is Not a Boundary Object: Reflections on the Origin of a Concept." Science, Technology, \& Human Values 35 (3): 601-617. https://doi.org/10.1177/0162243910377624

Star, Susan Leigh, and James R. Griesemer. 1989. "Institutional Ecology, 'Translations' and Boundary Objects: Amateurs and Professionals in Berkeley's Museum of Vertebrate Zoology, 1907-39." Social Studies of Science 19 (3): 387-420. https://doi.org/10.1177/030631289019003001 


\section{Biographies}

Teoma Naccarato and John MacCallum have been collaborating since they met in 2013. Their collective work draws on their disciplinary training in music composition, choreography, creative writing, and computer science, as well as their deep interest in performance art and philosophy. Together they devise performance-installations and publications, and guide workshops on the themes of 'Relational Listening' and on the 'critical appropriation of biosensors in artistic practice.' Teoma is currently completing her PhD at the Centre for Dance Research (C-DaRE) at Coventry University in the UK, and did her MFA at The Ohio State University, and her BFA at Concordia University in Montreal, all in dance/choreography. John did his PhD at UC Berkeley, his MM at McGill University in Montreal, and his BM at the University of the Pacific in California, all in music composition. They have each held a variety of research and teaching positions over the years in the US and Europe, and currently live in Berlin.

(c) 2019 John MacCallum and Teoma Naccarato

cc) (i) (2) Except where otherwise noted, this work is licensed under a Creative Commons Attributioncc) 